\title{
L'anémone*
}

\author{
Hélène Mino
}

\section{The Anemone}

Iso narrates her encounter with an other, her lover, a blue-eyed woman in the heat of the summer, their troubling relationship, and their parting in the cold of winter. Throughout the narration, the image of the sea-anemone clinging to a rock recurs, as metaphor of the relationship, its tenacity, its entanglements.

to D.R.

Je, Iso ne sait pas très bien comment j'en suis arrivée là. Je l'ai sans doute déjà su. C'était peut-être après un naufrage de bateau, ou une explosion nucléaire. Ce sont des évènements qui servent de points de repère, marquent le temps. Je crois que j'y suis surtout arrivée par hasard, comme toujours quand je marche. Il faisait chaud, très chaud. Il y avait peu d'ombre. Je marchais et l'asphalte était dure. C'était un hasard. Je l'ai reconnue, voilà tout.

Je me souviens très bien de ce premier hasard là et del'été chaud, de la route sans arbre au bord de la côte, des étoiles de nuit et de mer. Plus loin, l'été s'arrête. Je ne sais plus très bien comment j'en suis arrivée à rester là.

Le temps passe sur moi et me reprend mes illusions. Le temps s'écrase sur moi. Cette femme. Je la regardais, elle me laissait la regarder. Le leitmotive de mon regard posé sur elle.

\footnotetext{
* L'anémone' was written in January 86 in Vancouver. L'anémone' was read on the waves of radio centre ville, Montreal's cooperative radio in Montreal in October 86.
} 
Une fois, elle avait brusquement pris conscience de mon corps. Un instant, le bleu de ses iris m'avait transformée en tigresse qui fait rouler ses flancs dans les grandes herbes.

Je repense à ses yeux bleus posés sur moi, pour ne pas oublier ma presence.

Combien de temps peut-on rester ainsi, suspendue dans des zones non-dites? Combien de temps les côtes tendues en appel? Je me suis souvent demandée si la patience précède l'ennui. Chaque matin, le ciel comme une savane aride. Chaque matin, renouer avec la patience. Combien de temps peut on rester ainsi?

Je m'étend sur le plancher gris, au milieu du salon. Mes reins, mes fesses sentent les reflets de l'air qui passe. Je trouve son appartement très beau, à cause des couleurs. Il y a un carré de lumière dans la maison d'à côté, on peut le voir toute la nuit. Souvent elle spéculait sur ce carré de lumière crue sans store, sur notre voisin. Pour moi, c'était plutôt comme une lune, carrée toujours pleine. Ces détails m’atteignent à présent.

JE SUIS DEVENUE UNE ANEMONE. Une anémone folle collée au rocher. J'occupe l'espace indéfini. Son espace. A peine un peu en retrait. Je ne sais plus encore ce que j'attends au-delà de $l^{\prime}$ imperceptible. Je sais que je résiste. Parfois je n'engourdis. Souvent j'ai peur, mais je reste et c'est la douleur qui me prend et me broie le ventre.

Je me suis domptée à la patience. Il y a des patiences qu'on refait chaque matin. Je parle du moment ou toutes les raisons d'attendre sont mortes. Tenace, l'anémone s'accroche au rocher en dehors des circonstances, en dehors de toutes circonstances et de toutes raisons.

Bien sur je pensais à elle en regardant la mer. Je pensait à elle dans l'appartement. Cette femme. Je pensais en regardant la mer: 'Sous cette eau calme que rien ne fait sursauter, chaque vague existe à l'état de possible'.

Il est difficile de dire qui de nous deux a parlé la première. Je sais que la table était entre nous, la bouteille de vin, les assiettes sales. Je me souviens aussi d'avoir été déchiquetée. Pourtant mon visage n'a pas suivi la courbe de ses mots, tenace. Je suffoquais, la douleur entrait de partout. Elle s'expliquait avec une gêne qui baissait le regard. Les 
mots durs s'enfoncaient en moi. Je me demandais si elle savait, à propos de la douleur ... un peu au moins. La douleur brulait mon corps, la douleur hurlait à mes oreilles. Mes os craquaient sous le choc. Mais tout reflet de l'affolement disparaissait dans mes yeux: je lui souris même, comme si l'anémone n'avait aucune importance. Nous avons continué à vivre ensemble.

Plusieurs jours ont passé, des mois peut-être. 'Cela fait dix ans ou dix vies.' dit l'anémone. Tout est si flou dans ce temps évadé. Un rituel: mes mains pétrissent la chaire de son dos maléable. La chaire douce se détend, les nerfs reprennent leur place. Avec mes mains, je repousse ses tensions. Je repose son corps. Je repose son corps avec patience, la même patience. Ma tête dans le creux de son épaule cherche un abri, mais ne s'arrête pas. Elle soupire. Son soupire me remonte dans les doigts et jusqu' aux cou des. Je melaisse sculpter parsa respiration. Les pores de ma peau s'ouvrent sous l'émotion, je souris en la massant.

Je reconnais ses mouvements. Je sais que dans un instant elle aura repris son travail. Entièrement absorbée, ses doigts rapides, chaque geste précis, calculé. Avant qu'elle ne s'étire, je glisse mes lèvres dans le bas de son dos. Elle rit, se lève. Nous ne disons rien.

Ce soir, encore je suis venue la voir travailler. J'aime beaucoup regarder ses grosses mains rondes et rapides. Je sais que pendant que mon regard la suit et entrecoupe son image de souvenirs d'été, elle m'oublit, les yeux fixés sur l'écran.

Les anémones sont des êtres en partie plante et animal qui donnent l'impression de faire du ballet aquatique, de se laisser porter par les courants de la mer. En fait, malgré leur apparente légèreté, la délicatesse de leurs milliers de filaments ondulants, les anémones adhèrent aux rochers avec une force incroyable.

Les vitres épaisses de l'aquarium grossissaient leurs filaments souples. Elle était fascinée par leur couleur, je préfèrais leur tenacité. Elle se souvient sûrement de l'aquarium, bien que nous n'en ayons jamais reparlé.

En général, nous parlons peu des souvenirs de cet été là. Parfois, une phrase m'échappe, remonte à la surface. L'air est marin tout d'un coup. Nos souffles rhythmés deviennent le vent. Nous connaissons ces moments. Cette intensité encombrante et soudaine qui nous laisse aux aguets. Elle évite ces moments. Quand une phrase m'échappe, 
alors et malgré la censure, son echo me répond, puissant avec l'appui du regard: 'Je m'en souviens très bien.' Elle se souvient sûrement de l'aquarium et peut-être aussi des anémones.

Il est tard quand nous sortons dans la rue. Elle ne s'arrête pas, comme moi, pour sentir l'humidité épaisse de la nuit qui se plaque sur nous. Elle s'enfonce rapidement dans la nuit. Elle ne sait plus où elle va. Elle marche après avoir travaillé. Elle longe les fenêtres grises, change đe trottoir, évite le parc. Elle change d'idée, bifurque. Je la suis en silence, les mains dans les poches. Il est difficile de l'interrompre dans sa marche pour lui faire voir les lumières de Noël qui se perdent dans le brouillard. Cette femme, elle ne s'arrête jamais et on dirait qu'elle sait ou elle va.

Usure. Il y a ce froid qui souffle de plus en plus du fond de ma tête. Puis, mes yeux m'échappent. Ce regard. Des paysages entiers basculent et s'effondrent. Les frissons d'hiver m'écorchent la peau. A present, nous évitons les ponts dans nos marches au travers de la ville. Nous évitons les têtes à têtes. Au dedans de moi la patience a fait des éclas d'obus. Je ne lui ai pas dit, mais elle le sait. Je m'aggrippe au rebord de sa fenêtre par habitude. Je me perds, je l'ai perdue.

$C^{\prime}$ est peut-être dans une marche que nous avons reparlé de nous. Elle pleurait. Je l'ai vu au coin de mon regard. Ensuite elle m'a donné des chocolats.

Le glacier lèche les premiers rochers. L'anémone. Les souvenirs perdus de l'anémone. La douleur s'est faufilée, discrète entre les machoires serrées. Toute cette glace qui s'étend pour ne rien sentir. Les longs filaments de l'anémone comme des bras d'amoureuse l'appelaient, une nuit les bras de l'anémone sont morts de froid.

Une femme, yeux trop bleus, mains trop rondes, s'appuie à la fenêtre. Le paysage délicat de cette Asie, la calme. Elle respire la lumière mauve du soir. Puis elle entendle rire. La femme se retourne. Elle connait ce rire depuis longtemps, reconnait tout de suite le rire de l'anémone. Ce grand rire fou. La douleur toujours silencieuse. d'Iso.

La femme se rappelle. Elle ne savait pas selaisser troubler parle rire d'une femme. 


\section{$124 \cdot$ Tessera}

Des années plus tard une femme se retourne. Elle sait. Même en exil dans cette terre solitaire du Japon qu'elle a choisie, même auprès de ses amants, qu'elle ne choisit pas toujours, la présence de l'anémone ne la quitte pas. 